\title{
Applications of Nachbin's Theorem concerning Dense Subalgebras of Differentiable Functions
}

\author{
M.S. KASHIMOTO \\ Received on December 26, 2017 / Accepted on April 26, 2018
}

\begin{abstract}
In this paper, we give some applications of Nachbin's Theorem [4] to approximation and interpolation in the the space of all $k$ times continuously differentiable real functions on any open subset of the Euclidean space.
\end{abstract}

Keywords: Nachbin's theorem, approximation of differentiable functions, Stone-Weierstrass theorem, interpolation.

\section{INTRODUCTION}

Let $\Omega$ be an open subset of $\mathscr{R}^{p}$ and let $k$ be a nonnegative integer. We denote by $C^{k}(\Omega ; \mathscr{R})$ the algebra of all $k$ times continuously differentiable real functions on $\Omega$ and consider the compact open topology of order $k \tau_{u}^{k}$, that is, the topology of uniform convergence for the functions and all their partial derivatives up to the order $k$ on compact subsets of $\Omega$.

For a multi-index $\alpha=\left(\alpha_{1}, \cdots, \alpha_{p}\right) \in \mathscr{N}_{0}^{p}$ of non-negative integers, let $|\alpha|:=\alpha_{1}+\cdots+\alpha_{p}$ be the order of $\alpha, \alpha !:=\alpha_{1} ! \cdots \alpha_{p}$ !, and for $|\alpha| \leq p$ let $D^{\alpha}:=\partial^{|\alpha|} / \partial x_{1}^{\alpha_{1}} \cdots \partial x_{p}^{\alpha_{p}}$ represents the corresponding linear partial differential operator acting on $C^{k}(\Omega ; \mathscr{R})$.

The topology $\tau_{u}^{k}$ is generated by the semi-norms $\sigma_{k, \Gamma}$ given by

$$
\sigma_{k, \Gamma}(f)=\sum_{|\alpha| \leq k} \frac{1}{\alpha !} \sup \left\{\left|\left(D^{\alpha} f\right)(x)\right|: x \in \Gamma\right\} \quad \text { for all } f \in C^{k}(\Omega ; \mathscr{R}),
$$

where $\Gamma$ runs over all compact subsets of $\Omega$. By Proposition 3, p. 8 [5], $C^{k}(\Omega ; \mathscr{R})$ is a topological vector space with respect to this topology.

In 1949 Nachbin [4] established the following interesting characterization of dense subalgebras of the space $C^{k}(\Omega ; \mathscr{R})$.

*Corresponding author: Marcia Sayuri Kashimoto - E-mail: mskashim@gmail.com

Departamento de Matemática e Computação, IMC, UNIFEI, Universidade Federal de Itajubá, 37500-903 Itajubá, MG, Brasil. E-mail: kaxixi@unifei.edu.br; mskashim@gmail.com 
Theorem 1. (Nachbin) Let $\Omega$ be an open subset of $\mathscr{R}^{p}$ and $L$ be a subalgebra of $C^{k}(\Omega ; \mathscr{R})$. Then $L$ is dense in $C^{k}(\Omega ; \mathscr{R})$ if and only if the following conditions are satisfied:

(a) given $x, y \in \Omega$ with $x \neq y$, there exists $f \in L$ such that $f(x) \neq f(y)$;

(b) given $x \in \Omega$, there exists $f \in L$ such that $f(x) \neq 0$;

(c) given $x \in \Omega$ and $u \in \mathscr{R}^{p}$ with $u \neq \mathbf{0}$, there exists $f \in L$ such that $\frac{\partial f}{\partial u}(x) \neq 0$.

The proof of this result can be found in [3] and [4].

Our aim is to use Nachbin's theorem to give a proof of a density theorem and a simultaneous interpolation and approximation theorem in the space $C^{k}(\Omega ; \mathscr{R})$.

\section{THE RESULTS}

The Urysohn's Lemma ([2] p. 281) for differentiable functions is the main tool we employed in the next lemma.

Lemma 1. Let $\Omega$ be an open subset of $\mathscr{R}^{p}, w_{1}, \ldots, w_{m}$ distinct points in $\Omega$, and $y_{1}, \ldots, y_{m}$ distinct real numbers. If $L$ is a dense vector subspace of $C^{k}(\Omega ; \mathscr{R})$, then there exists a function $h \in L$ such that $h\left(w_{j}\right)=y_{j}, j=1, \ldots, m$.

Proof. Let $L$ be a dense linear subspace of $C^{k}(\Omega ; \mathscr{R})$ and $S=\left\{w_{1}, \ldots, w_{m}\right\}$ be a subset of $\Omega$. Consider the following linear mapping

$$
\begin{aligned}
T: C^{k}(\Omega ; \mathscr{R}) & \rightarrow \mathscr{R}^{m} \\
f & \mapsto\left(f\left(w_{1}\right), \ldots, f\left(w_{m}\right)\right) .
\end{aligned}
$$

Notice that $T$ is continuous.

For each $w_{i} \in S$ consider an open neighborhood $U_{i} \subset \Omega$ of $w_{i}$ such that $w_{j} \notin U_{i}$, for all $j \neq i, j \in$ $\{1, \ldots, m\}$. It follows from the Urysohn's Lemma for differentiable functions that there exists an infinitely differentiable function $\Phi_{i}: \mathscr{R}^{m} \rightarrow R, 0 \leq \Phi_{i} \leq 1$, such that $\Phi_{i}\left(w_{i}\right)=1$ and $\Phi_{i}(x)=0$, if $x \notin U_{i}$, in particular, $\Phi_{i}\left(w_{j}\right)=0, j \neq i$. Let $\phi_{i}=\left.\Phi_{i}\right|_{\Omega}$ the restriction of the function $\Phi_{i}$ to the subset $\Omega$ and $e_{i} \in \mathscr{R}^{m}$ the vector whose $i^{t h}$ coordinate is equal to 1 and the others are equal to 0 . The linear mapping $T$ is surjective since for any $\left(c_{1}, \ldots, c_{m}\right) \in \mathscr{R}^{m}$, we have

$$
\left(c_{1}, \ldots, c_{m}\right)=\sum_{i=1}^{m} c_{i} e_{i}=\sum_{i=1}^{m} c_{i}\left(\phi_{i}\left(w_{1}\right), \ldots, \phi_{i}\left(w_{m}\right)\right)=\sum_{i=1}^{m} c_{i} T\left(\phi_{i}\right)=T\left(\sum_{i=1}^{m} c_{i} \phi_{i}\right),
$$

where $\sum_{i=1}^{m} c_{i} \phi_{i} \in C^{k}(\Omega ; \mathscr{R})$. Moreover, $T(L)$ is closed because it is a linear subspace of $\mathscr{R}^{m}$. Then by density of $L$ and continuity of $T$, it follows that

$$
\mathscr{R}^{m}=T\left(C^{k}(\Omega ; \mathscr{R})\right)=T(\bar{L}) \subset \overline{T(L)}=T(L) .
$$


Therefore, for any $\left(y_{1}, \ldots, y_{m}\right) \in \mathscr{R}^{m}$ there exists $h \in L$ such that $T(h)=\left(y_{1}, \ldots, y_{m}\right)$, that is, $\left(h\left(w_{1}\right), \ldots, h\left(w_{m}\right)\right)=\left(y_{1}, \ldots, y_{m}\right)$.

We give a proof of the following density result.

Theorem 2. Let $V$ be an open subset of $\mathscr{R}^{p}, L$ a dense subalgebra of $C^{k}(V ; \mathscr{R})$, and $v_{1}, \ldots, v_{n}$ distinct points in $V$. Consider the open subset of $\mathscr{R}^{p}$,

$$
\Omega=V \backslash\left\{v_{1}, \ldots, v_{n}\right\}
$$

and the subalgebra

$$
M=\left\{\left.f\right|_{\Omega}: f \in L, f\left(v_{1}\right)=\ldots=f\left(v_{n}\right)=0\right\} .
$$

Then, $M$ is dense in $C^{k}(\Omega ; \mathscr{R})$.

Proof. Clearly $M$ is a subalgebra of $C^{k}(\Omega ; \mathscr{R})$. Let $x, y$ be any distinct points in $\Omega$. Consider the following subset

$$
S=\left\{x, y, v_{1}, \ldots, v_{n}\right\}
$$

of $V$. By Lemma 1 there exists $h \in L$ such that $h(x)=1, h(y)=-1$ and $h\left(v_{j}\right)=0$ for $j=1, \ldots, n$. Then, $\left.h\right|_{\Omega} \in M$ and satisfies Conditions (a) and (b) of Theorem 1.

Now let $z \in \Omega$ and $u \in \mathscr{R}^{p}, u \neq \mathbf{0}$. It follows from Lemma 1 that there exists $g \in L$ such that $g(z)=1$ and $g\left(v_{j}\right)=0$ for $j=1, \ldots, n$. Hence, $\left.g\right|_{\Omega} \in M$. If $\frac{\partial g}{\partial u}(z) \neq 0$ the Condition (c) of Theorem 1 is satisfied. Otherwise, notice that $L$ is not a subset of

$$
B=\left\{f \in C^{k}(V ; \mathscr{R}): \frac{\partial f}{\partial u}(z)=0\right\},
$$

since $L$ is a dense subalgebra of $C^{k}(V ; \mathscr{R})$ and $B$ is a proper closed subalgebra of $C^{k}(V ; \mathscr{R})$. Thus, there exists $\phi \in L$ such that $\frac{\partial \phi}{\partial u}(z) \neq 0$. Then, $\phi g \in L$ and $\phi g\left(v_{j}\right)=0$ for $j=1, \ldots, n$, that is, $\left.\phi g\right|_{\Omega} \in M$. Moreover,

$$
\frac{\partial \phi g}{\partial u}(z)=\frac{\partial \phi}{\partial u}(z) g(z)+\phi(z) \frac{\partial g}{\partial u}(z)=\frac{\partial \phi}{\partial u}(z) g(z)=\frac{\partial \phi}{\partial u}(z) \neq 0 .
$$

Thus, by Theorem $1, M$ is dense in $C^{k}(\Omega ; \mathscr{R})$.

For each positive integer $l, \mathscr{P}^{l}\left(\mathscr{R}^{p}, \mathscr{R}\right)$ denotes the linear subspace of $C^{k}\left(\mathscr{R}^{p} ; \mathscr{R}\right)$ generated by the set of all functions of the form

$$
p(x)=[\psi(x)]^{l}, \quad x \in \mathscr{R}^{p},
$$

where $\psi \in\left(\mathscr{R}^{p}\right)^{*}$, the dual space of $\mathscr{R}^{p}$. The elements of $\mathscr{P}^{l}\left(\mathscr{R}^{p}, \mathscr{R}\right)$ are called the $l$ homogeneous continuous polynomials of finite type from $\mathscr{R}^{p}$ into $\mathscr{R}$. The subspace of $C^{k}\left(\mathscr{R}^{p} ; \mathscr{R}\right)$ consisting of all functions of the form 


$$
p(x)=p_{0}+\sum_{j=1}^{l} p_{j}(x), \quad x \in \mathscr{R}^{p}
$$

where $p_{0} \in \mathscr{R}, p_{j} \in \mathscr{P}^{j}\left(\mathscr{R}^{p}, \mathscr{R}\right), j=1, \ldots, l, l \in \mathscr{N}$, is denoted by $\mathscr{P}\left(\mathscr{R}^{p}, \mathscr{R}\right)$. Its elements are called real continuous polynomials of finite type. The polarization formula shows that $\mathscr{P}\left(\mathscr{R}^{p}, \mathscr{R}\right)$ is a subalgebra of $C^{k}\left(\mathscr{R}^{p} ; \mathscr{R}\right)$. Indeed, given $\psi_{1}$ and $\psi_{2}$ in $\left(\mathscr{R}^{p}\right)^{*}$,

$$
\psi_{1}(x) \psi_{2}(x)=\frac{1}{4}\left[\left(\psi_{1}(x)+\psi_{2}(x)\right)^{2}-\left(\psi_{1}(x)-\psi_{2}(x)\right)^{2}\right]
$$

shows that $\psi_{1} \psi_{2} \in \mathscr{P}^{2}\left(\mathscr{R}^{p}, \mathscr{R}\right)$, since $\psi_{1}+\psi_{2}$ and $\psi_{1}-\psi_{2}$ belong to $\left(\mathscr{R}^{p}\right)^{*}$.

Corollary 3. Let $v_{1}, \ldots, v_{n}$ be distinct points in $\mathscr{R}^{p}$. Consider the open subset of $\mathscr{R}^{p}$,

$$
\Omega=\mathscr{R}^{p} \backslash\left\{v_{1}, \ldots, v_{n}\right\}
$$

and the subalgebra

$$
M=\left\{\left.f\right|_{\Omega}: f \in \mathscr{P}\left(\mathscr{R}^{p} ; \mathscr{R}\right), f\left(v_{1}\right)=\ldots=f\left(v_{n}\right)=0\right\}
$$

Then, $M$ is dense in $C^{k}(\Omega ; \mathscr{R})$.

Proof. First of all, we verify that the subalgebra $P\left(\mathscr{R}^{p} ; \mathscr{R}\right)$ is dense in $C^{k}\left(\mathscr{R}^{p} ; \mathscr{R}\right)$. Given $x, y \in \mathscr{R}^{p}$ with $x \neq y$, it follows from Hahn-Banach Theorem that there exists $\psi \in\left(\mathscr{R}^{p}\right)^{*}$ such that $\psi(x) \neq \psi(y)$. Since $\left(\mathscr{R}^{p}\right)^{*}=P^{1}\left(\mathscr{R}^{p} ; \mathscr{R}\right) \subset P\left(\mathscr{R}^{p} ; \mathscr{R}\right)$, the Condition (a) of Theorem 1 is satisfied. By definition, $P\left(\mathscr{R}^{p} ; \mathscr{R}\right)$ contains all the constant functions. Now, let $\mathbf{0} \neq u=\left(u_{1}, \ldots, u_{p}\right) \in$ $\mathscr{R}^{p}$. Then, there exists $0 \neq u_{j} \in \mathscr{R}, j \in\{1, \ldots, p\}$. Let $\Pi_{j}: \mathscr{R}^{p} \rightarrow \mathscr{R}$ defined by $\Pi_{j}(x)=x_{j}$, $x \in \mathscr{R}^{p}$. Since $\frac{\partial \Pi_{j}}{\partial x_{j}}(x)=1$ and $\frac{\partial \Pi_{j}}{\partial x_{i}}(x)=0$ for $i \neq j$, it follows that

$$
\frac{\partial \Pi_{j}}{\partial u}(x)=\sum_{i=1}^{p} u_{i} \frac{\partial \Pi_{j}}{\partial x_{i}}(x)=u_{j} \neq 0 .
$$

Therefore, by Theorem $1, P\left(\mathscr{R}^{p} ; \mathscr{R}\right)$ is dense in $C^{k}\left(\mathscr{R}^{p} ; \mathscr{R}\right)$ and the assertion follows from Theorem 2.

Motivated by an extended Stone-Weierstrass theorem (see Corollary 1.1 [1]), we give a proof of a result concerning simultaneous interpolation and approximation in $C^{k}(\Omega ; \mathscr{R})$. The tools are the Nachbin's Theorem and the following result due to Deutsch.

Theorem 4. (Deutsch) Let $Y$ be a dense vector subspace of the topological vector space $Z$ and let $T_{1}, \ldots, T_{n}$ be continuous linear functionals on $Z$. Then for each $f \in Z$ and each neighborhood $U$ of $f$ there is $y \in Y$ such that $y \in U$ and $T_{i}(y)=T_{i}(f), i=1, \ldots, n$.

Theorem 5. Let $\Omega$ be an open subset of $\mathscr{R}^{p}, x_{1}, \ldots, x_{n}$ distinct elements of $\Omega$ and L a subalgebra of $C^{k}(\Omega ; \mathscr{R})$ that satisfies the following conditions, 
(a) given $x, y \in \Omega$ with $x \neq y$, there exists $f \in L$ such that $f(x) \neq f(y)$;

(b) given $x \in \Omega$, there exists $f \in L$ such that $f(x) \neq 0$;

(c) given $x \in \Omega$ and $u \in \mathscr{R}^{p}$ with $u \neq \mathbf{0}$, there exists $f \in L$ such that $\frac{\partial f}{\partial u}(x) \neq 0$.

Then, for each $f \in C^{k}(\Omega ; \mathscr{R})$, and each neighborhood $U$ of $f$ there exists $g \in L \cap U$ such that $f\left(x_{i}\right)=g\left(x_{i}\right)$ for $i=1, \ldots, n$.

Proof. It follows from Theorem 1 that $L$ is a dense subalgebra of the topological vector space $C^{k}(\Omega ; \mathscr{R})$. Let $S=\left\{x_{1}, \ldots, x_{n}\right\} \subset \Omega$. Notice that

$$
\begin{aligned}
T_{i}: C^{k}(\Omega ; \mathscr{R}) & \rightarrow \mathscr{R} \\
f & \mapsto f\left(x_{i}\right)
\end{aligned}
$$

is a continuous linear functional for each $i=1, \cdots, n$. Setting $Z=C^{k}(\Omega ; \mathscr{R})$ and $Y=L$, the conclusion follows from Theorem 4 .

\section{ACKNOWLEDGEMENTS}

The author acknowledges the referees for the valuable comments and suggestions which improved the presentation of the paper.

RESUMO. Em 1949, Leopoldo Nachbin estabeleceu uma versão do Teorema de StoneWeierstrass para funções diferenciáveis de classe $C^{k} \mathrm{em}$ abertos do espaço euclidiano. Neste trabalho, apresentamos algumas aplicacões desse teorema relacionadas com aproximação e interpolação no espaço das funções de classe $C^{k}$ munido da topologia compacto-aberta.

Palavras-chave: Teorema de Nachbin, aproximação de funções diferenciáveis, Teorema de Stone-Weierstrass, interpolação.

\section{REFERENCES}

[1] F. Deutsch, Simultaneous interpolation and approximation in linear topological spaces. SIAM J. Appl. Math., 14 (1966), 1180-1190.

[2] M. Moskowitz \& F. Paliogiannis, "Functions of Several Real Variables". World Scientific, Singapore (2011).

[3] J. Mujica, Subálgebras densas de funciones diferenciables. Cubo Matemática Educacional, 3 (2001), $121-128$.

[4] L. Nachbin, Sur les algèbres denses de fonctions différentiables sur une variété. Comptes Rendus de l'Académie des Sciences de Paris, 228 (1949), 1549-1551.

[5] L. Nachbin, "Elements of Approximation Theory". Van Nostrand, Princeton, NJ (1967), reprinted by Krieger, Huntington, NY (1976). 